\title{
MDM2 mediates p73 ubiquitination: a new molecular mechanism for suppression of p73 function
}

\author{
Hong $\mathbf{W u}^{1}$, Roger P. Leng ${ }^{1}$ \\ ${ }^{1}$ Department of Laboratory Medicine and Pathology, University of Alberta, Edmonton, Alberta, Canada \\ Correspondence to: \\ Hong Wu, e-mail: hwu8@ualberta.ca \\ Roger P. Leng, e-mail: rleng@ualberta.ca \\ Keywords: ubiquitin E3 ligase, ubiquitination, tumor suppressor, oncogene, protein degradation \\ Received: March 11, $2015 \quad$ Accepted:May 14, $2015 \quad$ Published: May 26, 2015
}

\section{ABSTRACT}

The protein p73, a homologue of the tumor suppressor protein p53, is capable of inducing apoptosis and cell cycle arrest. MDM2 is transcriptionally activated by p73 and represses the functions of p73, including p73-dependent transactivation and growth suppression. However, the molecular mechanism of this repression is unknown. In this study, we show that MDM2 mediates p73 ubiquitination. MDM2 mainly utilizes K11, K29 and K63-linked chains to mediate p73 ubiquitination in vivo and in vitro. However, MDM2 is unable to promote p73 degradation in most tested cell lines. Surprisingly, we observe that overexpression of Mdm2 promotes p73 degradation mainly through Itch in Mdm2-null MEFs. We further find that Itch interacts with the transfected Mdm2 in Mdm2-null cells. Moreover, our findings reveal that the E3 ligase activity of MDM2 is required to repress p73-dependent apoptosis and cell cycle arrest but not p73-dependent transcriptional activity. Furthermore, the data suggest a link between p73 ubiquitination/MDM2 E3 ligase activity and p73 biological functions.

\section{INTRODUCTION}

The protein $\mathrm{p} 73$, which is a homologue of $\mathrm{p} 53$, is capable of inducing apoptosis or cell cycle arrest [1-4]. Although p73 has apoptotic activity, it is rarely mutated in human tumors, and p73 deficient mice do not have an increase in tumor incidence [1]. The literature describing the tumor-suppressor function of $\mathrm{p} 73$ is complex and controversial, and the role of $\mathrm{p} 73$ has been a matter of debate. However, a number of studies have recently shown that $\mathrm{p} 73$ expression is lost or reduced in certain human tumors (loss of heterozygosity, allele silencing, etc.), indicating that $\mathrm{p} 73$ has tumor-suppressing activities [5-7]. Mice that are heterozygous for $\mathrm{p} 73$ develop spontaneous tumors; the loss of p73 can cooperate with p53 in tumor suppression; and tandem mutation of p73 and p53 leads to a more aggressive tumor phenotype. These findings indicate that p73 plays an important role in tumor suppression in mice [8]. Itch was identified as an E3 ligase for p73 [9]; however, multiple pathways may be involved in the control of $\mathrm{p} 73$ functions.

Mdm2 was originally identified as an amplified gene in a spontaneously transformed derivative of mouse BALB/c cells [10]. Mdm2 (known as MDM2 or $\mathrm{Hdm} 2$ in humans) interacts with the p53 transactivation domain $[11,12]$ and promotes p53 degradation via the ubiquitinproteasome pathway [13-15]. The deletion of Mdm2 in mice results in an embryonic lethal phenotype, but lethality can be prevented by deletion of the p53 gene, indicating the importance of the negative regulatory function of Mdm2 on p53 during development [16, 17]. Interestingly, MDM2 is transcriptionally activated by $\mathrm{p} 73$ and represses many of its functions, such as p73-dependent transactivation and growth suppression $[18,19]$. However, the molecular mechanisms of this repression are unknown.

In this study, we observe that MDM2 promotes $\mathrm{p} 73$ ubiquitination. We show that MDM2 is required to mediate p73 ubiquitination in vivo. MDM2 is unable to promote p73 degradation in most tested cell lines. However, our findings reveal that overexpression of $\mathrm{Mdm} 2$ promotes p73 degradation in Mdm2-null MEFs, and is dependent on Itch. Depletion of MDM2 by siRNA activates p73dependent transactivation and apoptosis. In addition, the RING domain of MDM2 is essential for MDM2-mediated p73 ubiquitination. 


\section{RESULTS}

\section{Overexpression of MDM2 mediates the ubiquitination of $\mathrm{p} 73$ in vivo}

MDM2 is a ubiquitin E3 ligase for p53 and promotes p53 degradation [15, 23, 24]. MDM2 has been reported to bind p73 and suppress its function without inducing degradation $[18,19]$. We investigated whether ectopic expression of MDM2 promotes $\mathrm{p} 73$ ubiquitination in vivo. Accordingly, p53-negative Saos-2 cells were transfected with expression plasmids encoding $\mathrm{p} 73 \alpha$ or $\mathrm{p} 73 \beta$ alone or with MDM2, MDM2 $\triangle$ RING (in which the RING domain has been deleted), ITCH, or an ITCH HECT mutant. In addition, cells were cotransfected with Myc-tagged wildtype ubiquitin (Ubwt) or Myc-tagged Ub4KR as indicated. $\mathrm{Ub} 4 \mathrm{KR}$ is a partial polyubiquitination-defective mutant (in which Lys11, Lys29, Lys48, and Lys 63 were each replaced by arginine, kindly provided by Dr. Y. Yarden). In vivo ubiquitination assays were performed under denaturing conditions [20-22]. Immunoprecipitation of p73 with a Flag-specific antibody (M5) was followed by immunoblotting with a Myc-specific antibody to detect ubiquitinated p73 (Figure 1A, 1B, upper image) or a p73specific antibody (ER-15) to detect all p73 (Figure 1A, 1B, lower image). Immunoprecipitated p73 (or proteins associated with $\mathrm{p} 73$ ) were heavily ubiquitinated in the presence of MDM2 and wild-type ubiquitin (Ubwt) (Figure 1A, 1B, upper image). However, MDM2 $\triangle \mathrm{RING}$ was unable to promote $\mathrm{p} 73$ ubiquitination, indicating that the RING domain of MDM2 is essential for its E3 ligase activity (Figure 1A, 1B). As shown in Figure 1A and 1B, Ub4KR results in significantly reduced MDM2-mediated p73 ubiquitination compared to Ubwt, suggesting that MDM2-mediated p73 ubiquitination occurs predominantly on Lys11, and/or Lys29, and/or Lys48, and/or Lys63 of ubiquitin in vivo. Saos-2 cells coexpressing Flag-p73 and MDM2 along with the Myc-tagged Ub4KR mutant recapitulate the Ubwt pattern; however, the levels of p73 ubiquitination are markedly reduced (Figure 1A, 1B). Consistent with previous reports $[18,19]$, MDM2 is unable to decrease the levels of $\mathrm{p} 73 \alpha$ and $\mathrm{p} 73 \beta$ proteins in Saos2 cells (third images, Figure 1A, 1B). These data indicate that MDM2 promotes predominantly multiple or polyubiquitination of $\mathrm{p} 73$ in vivo. These findings support the hypothesis that MDM2 plays an important role in promoting p73 ubiquitination. Similar results were obtained using H1299 cells (Supplementary Figure S1). It was reported that Itch is an E3 ligase for p73 that negatively regulates p73 stability [9]. We overexpressed MDM2 and ITCH/ AIP4 (the human homolog of mouse Itch) in Saos-2 cells using plasmids expressing Ubwt or the Ub4KR mutant to mediate ubiquitination of p73. Notably, we detected weak p73 ubiquitination in the ITCHC830A mutant, suggesting that this point mutant of Itch may have weak E3 ligase activity (Figure 1A, 1B). To identify the lysine residue(s) of ubiquitin $(\mathrm{Ub})$ required for p73 ubiquitination by MDM2 in vivo, we examined a number of Ub mutants. The mutants each contained one lysine, with the remaining five lysine residues (K6, K11, K29, K48, and K63) mutated to arginine. $p 73 \alpha$ or $p 73 \beta$ and HA-tagged ubiquitin or various HA-tagged ubiquitin mutants were coexpressed with MDM2 in Saos-2 cells. As shown in Figure 1C, our finding suggested that MDM2 may preferentially utilize multiple Lys residues of ubiquitin to promote $\mathrm{p} 73$ ubiquitination in vivo. The p73 immunoblots revealed that MDM2 promotes polyubiquitination of $\mathrm{p} 73$ in vivo (Figure 1C, lower image). To provide direct evidence that the modified p73 species corresponds to ubiquitin conjugation, we coexpressed His-tagged ubiquitin and $\mathrm{p} 73 \alpha$ or $\mathrm{p} 73 \beta$ with or without MDM2 or ITCH in Saos-2 cells and isolated His-ubiquitin conjugated proteins under denaturing conditions. Ubiquitin conjugation was detected in the presence of MDM2, as did the coexpression of p73 and ITCH (Figure 1D). Taken together, these data demonstrate that MDM2 promotes $\mathrm{p} 73$ ubiquitination in vivo.

\section{MDM2 is required for $\mathrm{p} 73$ ubiquitination in vivo}

To determine whether MDM2 functions as an E3 ligase for $\mathrm{p} 73$ in vivo, HEK293 cells were transfected with control-siRNA or MDM2-siRNA constructs. After 40 hours, the cells were transfected with an HA-tagged ubiquitin expression plasmid and immunoprecipitated with a p73specific antibody (ER-15) under denaturing conditions. Ubiquitination of p 73 was markedly decreased upon MDM2-siRNA treatment compared with controlsiRNA treatment (Figure 2A). Stronger ubiquitination of p73 was observed when cells were treated with the proteasome inhibitor MG132, suggesting that MG132 treatment can increase ubiquitinated p73 levels (Figure 2B). Similar results were observed in Mdm2- mouse embryo fibroblasts (MEFs) expressing HA-tagged ubiquitin (Figure 2C). p73 (or proteins that coimmunoprecipitation with p73) appeared heavily ubiquitinated when Mdm2 was reintroduced into Mdm2 null MEFs (Figure 2C, lane 3, upper image) and the levels of p73 proteins were lower compared to that of wild type MEFs or Mdm2 null MEFs (Figure 2C, lower image). The p73 immunoblot revealed that mono- or multiple-ubiquitinated p73 occurred to a lesser extent in MDM2-siRNA than in control-siRNA cell lines, indicating an inverse correlation between the poly-and the mono-form of $\mathrm{p} 73$ (Figure 2A-2C, lower image). The levels of p 73 did not change significantly when HEK293 cells were treated with MDM2-siRNA (Figure 2A, 2B), consistent with previous reports [18, 19]. These data indicate that MDM2 is required for $\mathrm{p} 73$ ubiquitination in vivo. To determine whether endogenous p73 and MDM2 could interact under more physiological conditions in the absence of overexpression, an IP/Western blotting experiment was performed using extracts prepared 
A

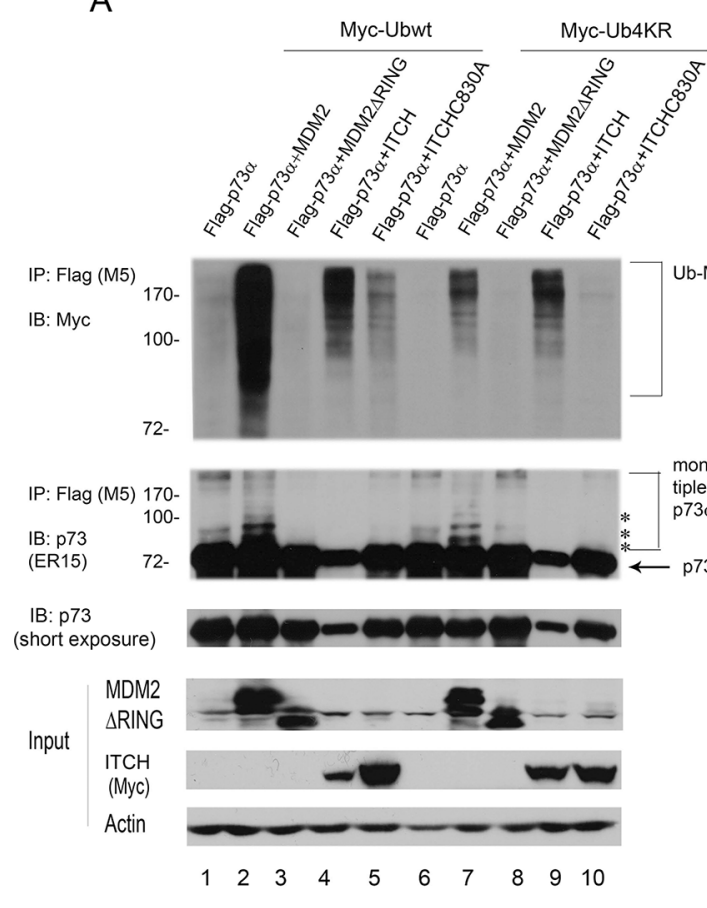

C

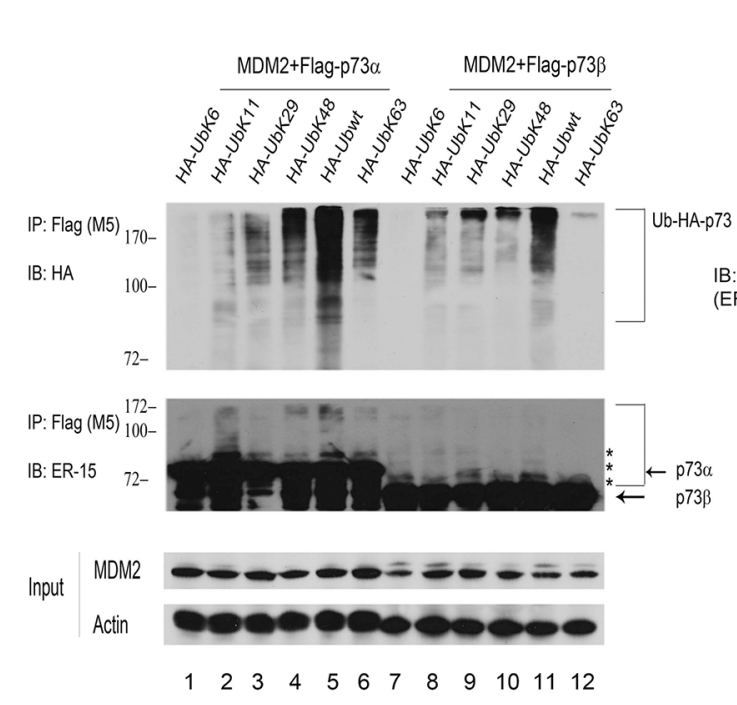

B
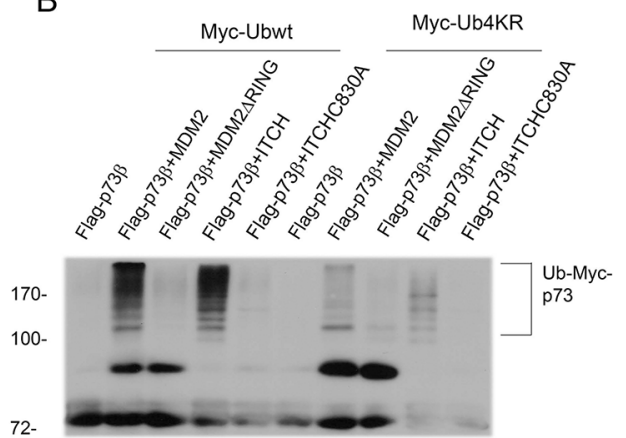

Input

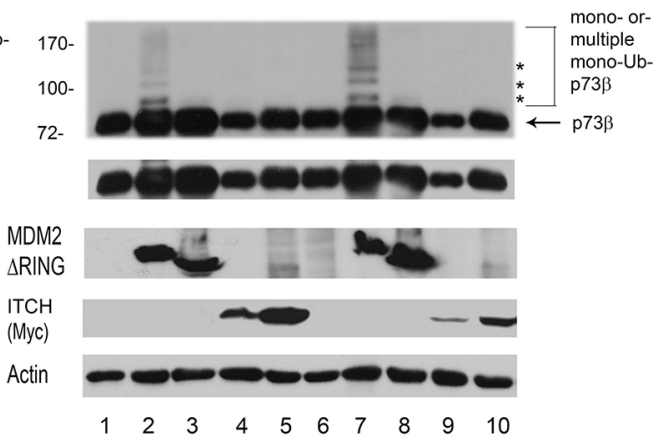

D

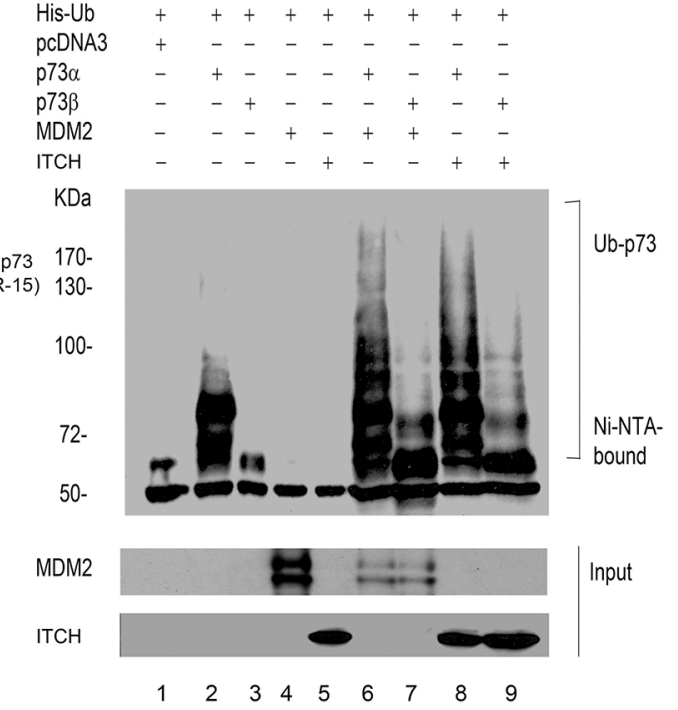

Figure 1: MDM2 promotes p73 ubiquitination in vivo. A. Saos-2 cells were cotransfected with plasmids expressing Flag-p $73 \alpha$ and pCMV-Bam-MDM2, MDM2 $\triangle$ RING, ITCH, or ITCH mutant (C830A) as well as Myc-Ubwt or Myc-Ub4KR as indicated. p73 was immunoprecipitated with a Flag-specific antibody (M5) and analyzed by immunoblotting with a Myc-specific antibody (upper image) and ER-15 for p73 (second image). Direct western blots for MDM2 and ITCH are shown in the lower panels. B. The same procedure as (A) was used, except that Saos-2 cells were cotransfected with a Flag-p73 $\beta$-expressing plasmid instead of Flag-p $73 \alpha$. C. The same procedure as (A) was used, except that Saos-2 cells were cotransfected with plasmids encoding p $73 \alpha$ or p $73 \beta$ and MDM2 along with a number of ubiquitin mutants as indicated. Asterisk (*) indicates the migration position of p73-Ub conjugates. D. Saos-2 cells were transfected with plasmids expressing His-ubiquitin, p73 $\alpha$ or p73 $\beta$, and MDM2 or ITCH. His-ubiquitinated proteins were isolated from denatured whole extract extracts, and analyzed by western blot with a p73 specific antibody (ER-15). Direct western blots for MDM2 and ITCH are shown in the lower panels.

from human HEK293 cells. We observed that (i) p73 was present in the anti-MDM2 immunoprecipitates, but not in the control mouse IgG; and (ii) MDM2 was present in the anti-p73 immunoprecipitates, but not in the control mouse $\mathrm{IgG}$; indicating that $\mathrm{p} 73$ and MDM2 physically interacts in vivo (Figure 2D). 
A

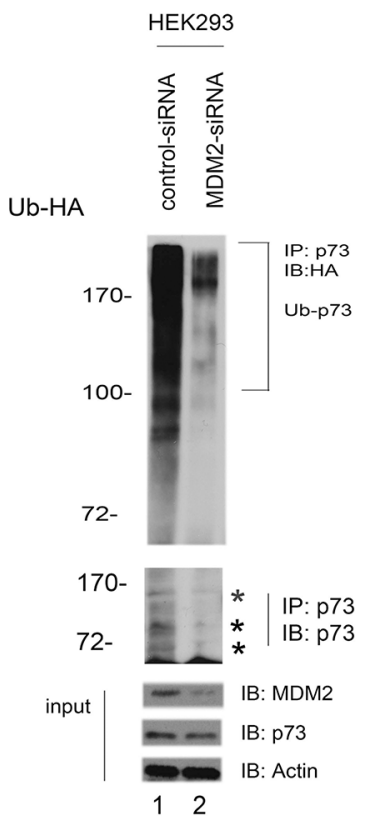

C
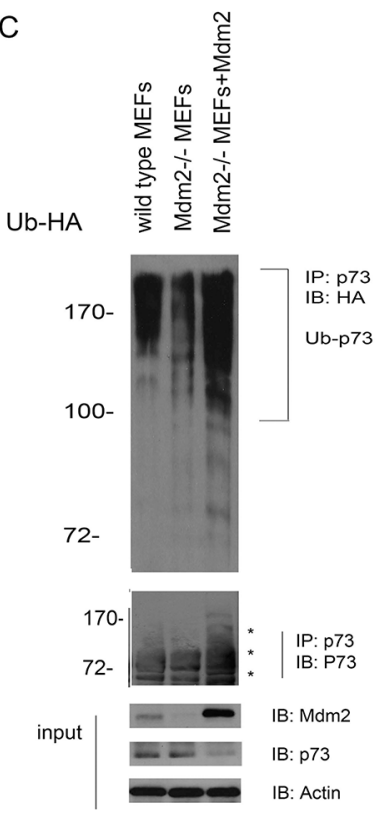

123
B
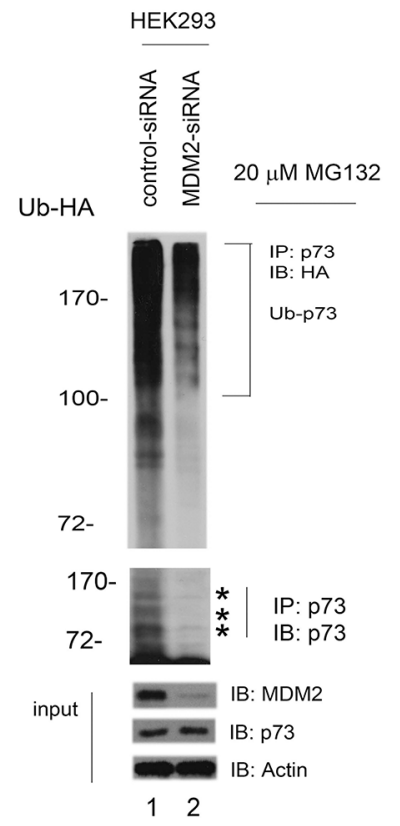

D

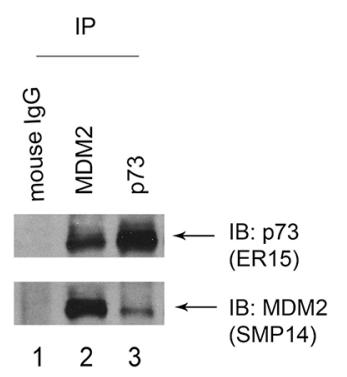

Figure 2: MDM2 is required for p73 ubiquitination in vivo. A. HEK293 cells were transfected with MDM2-siRNA or control-siRNA constructs. Forty hours later, the cells were further transfected with a plasmid expressing HA-Ub. Lysates were immunoprecipitated with a p73specific antibody (ER-15) and analyzed by western blotting with an HA-specific antibody to detect ubiquitinated p73. The western blots for p73, MDM2, and actin are shown in the lower panel. B. The same procedure as (A) was used, except that after the second transfection with the HA-Ub expression plasmid, the cells were treated with the proteasome inhibitor MG132 $(20 \mu \mathrm{M}) 6 \mathrm{hr}$ prior to harvest. C. Similar to (A) except that the cell extracts were obtained from the wild-type mouse embryonic fibroblasts (MEFs) and Mdm2 null MEFs. In addition, Mdm2 expression plasmid was reintroduced into Mdm2 null MEFs (lane 3). D. HEK293 cells were immunoprecipitated with anti-p73 (ER-15) or anti-MDM2 (SMP14) as indicated, and immunoblotted with antip73 (upper image, ER-15) and anti-MDM2 antibodies (lower image). 


\section{MDM2 promotes $\mathrm{p} 73$ ubiquitination in vitro}

The experiments described above were performed in living cells, which contain endogenous E1, E2, E3, and E4 enzymes. To determine if p73 could serve as a substrate for MDM2-mediated ubiquitination in vitro, purified His-p73 $\alpha$ or His-p73 $\beta$ was incubated with GST-MDM2 and Ubwt. As shown in Figure 3A and 3B, MDM2 promotes $\mathrm{p} 73$ ubiquitination dependently of the presence of E1, E2, and Ub. To further evaluate above results, a series of ubiquitin mutants that contain one lysine with the remaining six lysine residues mutated to arginine (K6, K11, K29, K48, K63) were generated, purified from Escherichia coli, and used for in vitro ubiquitination assay. In the presence of MDM2 and p73, we detected high levels of Ub-Lys11, Lys29, wt-Ub conjugation, and moderate levels of Ub-Lys6, 48, 63 conjugation (Figure 3C, upper image). The p73 immunoblots reveal that there are different patterns of ubiquitination between $\mathrm{p} 73 \alpha$ and $\mathrm{p} 73 \beta$, suggesting that MDM2 may utilize Lys11 and Lys29 of Ub to promote the ubiquitination of $\mathrm{p} 73 \alpha$; by contrast, MDM2 may utilize multiple residues of $\mathrm{Ub}$ to mediate $\mathrm{p} 73 \beta$ ubiquitination in vitro (Figure 3C, lower image). To eliminate possible autoubiquitination of MDM2, we performed coupled in vitro ubiquitination/IP. After a 1-hr reaction, the mixtures were immunoprecipitated with a p73-specific antibody (ER-15) and analyzed by immunoblotting with an anti-Ub monoclonal antibody to detect ubiquitinated p73 (Figure 3D, upper image), ER-15 to detect total p73 (Figure 3D, middle image), FK-1 to detect polyubiquitination of p73 (Figure 3D, third image), and anti-ubiquitin, Lys63specific and Lys48-specific antibodies to detect Lys63 or Lys48-linked polyubiquitination of p73 (Figure 3D, lower image). Notably, p73 is polyubiquitinated by MDM2 in the presence of Ubwt and to a lesser extent in the presence of Lys63-linked chains for $\mathrm{p} 73 \alpha$ or $\mathrm{p} 73 \beta$; but not recognize by the Lys48-specific antibody (Figure 3D, lower image). These data indicate that MDM2 mainly utilizes Lys11, Lys 29 and Lys63 to mediate $\mathrm{p} 73$ ubiquitination in vitro. It has been reported that low level of MDM2 induces monoubiquitination of $\mathrm{p} 53$, whereas high level of MDM2 promotes polyubiquitination of $\mathrm{p} 53$ [25]. We investigated whether $\mathrm{p} 73$, the structural and functional homologue of $\mathrm{p} 53$, can be regulated by different levels of MDM2 in vitro. As shown in Figure 3E (upper image), polyubiquitination of p73 was detected in the presence of high level of MDM2 with Ubwt but not UbKO. When analyzed with FK1 (Figure 3E, lower image), polyubiquitination of $\mathrm{p} 73$ was detected. These data suggest that like p53, p73 polyubiquitination mediated by MDM2 is dependently of the level of MDM2. Consistently, we observed that the ITCH-C830A has weak E3 ligase activity compared to that of ITCH $\triangle$ HECT in vitro (Figure 3 F). There are several cysteine residues in the HECT domain, it is possible that under certain circumstances they can serve as ubiquitin acceptors. An important consideration is why MDM2 is unable to promote p73 degradation in HEK293 cells. A recent study suggested that proteasomal degradation of some proteins requires 2 binding interactions, including polyubiquitin chains and an intrinsic proteasomal binding element in the substrates [26]. However, that study did not identify the proteasomal binding element. It is possible that the intrinsic binding element in p73 is inactive or unable to bind to the $26 \mathrm{~S}$ proteasome. It is also possible that polyubiquitination of $\mathrm{p} 73$ by MDM2 primarily utilizes Lys11 or Lys29 of ubiquitin but not Lys48 (Figure 3D, lower image). Therefore, the mechanism by which MDM2 mediates p73 polyubiquitination without affecting its stability need to be further investigated.

\section{Overexpression of $\mathrm{Mdm} 2$ promotes p73 degradation in Mdm2-null MEFs}

Itch binds to $\mathrm{p} 73$ and promotes $\mathrm{p} 73$ ubiquitination and degradation [9]. We therefore examined whether Itch mediates p73 ubiquitination through Mdm2. Mdm2 $2^{-/}$p5 $3^{--}$double null MEFs were transfected with plasmids expressing p $73 \alpha$ alone or with Itch. A plasmid expressing the enhanced GFP (pEGFP) was used for measuring transfection efficiency. Extracts were prepared and analyzed by western blotting with a p73-specific antibody (H-79) and a Myc-specific antibody for Itch. As shown in Figure 4A, overexpression of Itch decreased the levels of p73 in Mdm2 null MEFs. To clarify the relationship between Itch and Mdm2, Mdm2 null MEFs were coexpressed with plasmids expressing p $73 \alpha$ alone or with Itch or both Itch and Mdm2, and analyzed by western blotting. Coexpression of Itch and Mdm2 further increased the degradation of $\mathrm{p} 73 \alpha$ (Figure 4B), suggesting that Mdm2 enhances Itch-mediated p 73 degradation. To investigate whether Itch is required for the Mdm2-mediated degradation of 73 in vivo, Mdm2-null MEFs were transfected with Itch-siRNA or control siRNA. Two days later, these cells were further transfected with the Mdm2 expression plasmid and subsequently analyzed by western blot. The overexpression of Mdm2 slightly affects $\mathrm{p} 73$ protein levels when Itch production in these cells was eliminated using siRNA (Figure 4C, 4D). This suggests that Itch is essential for Mdm2-mediated p73 degradation in Mdm2-null MEFs. To determine whether overexpression of $\mathrm{Mdm} 2$ regulates the level of endogenous p73 protein, Mdm2-null cells were transfected with increasing amounts of Mdm2 expression plasmid. The transient overexpression of Mdm2 decreased the levels of endogenous $\mathrm{p} 73$ protein (Figure 4E), suggesting that the $\mathrm{p} 73$ proteins are target of $\mathrm{Mdm} 2$. 
A

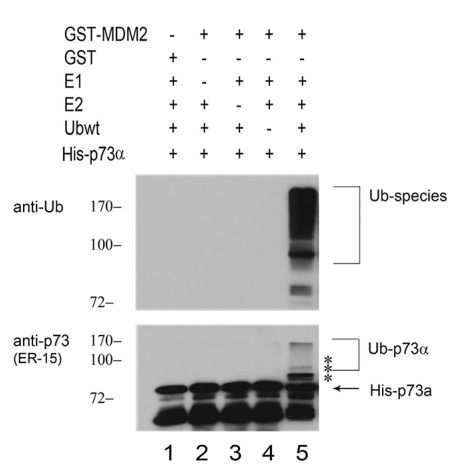

C

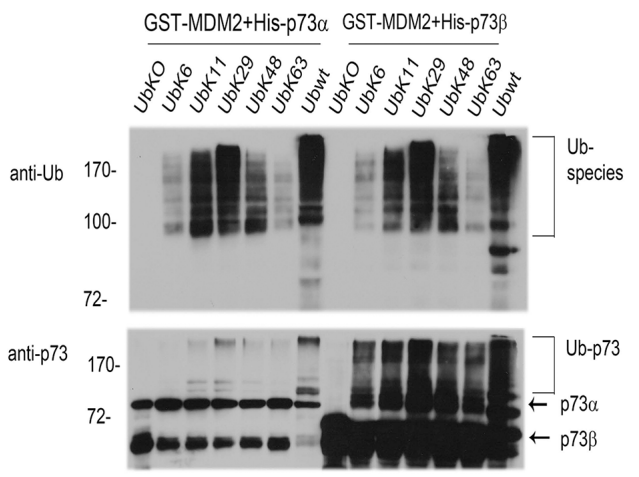

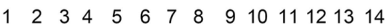

E

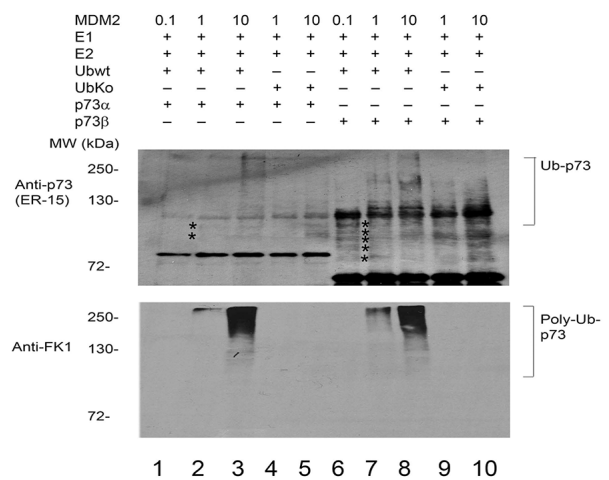

B

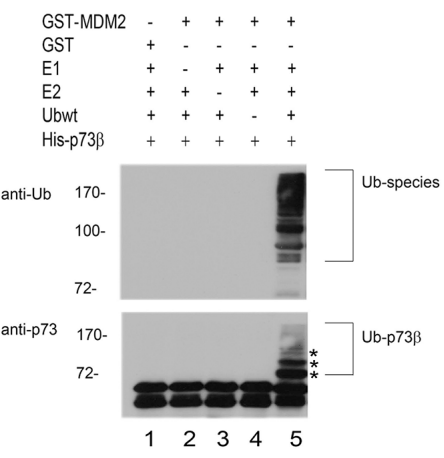

D

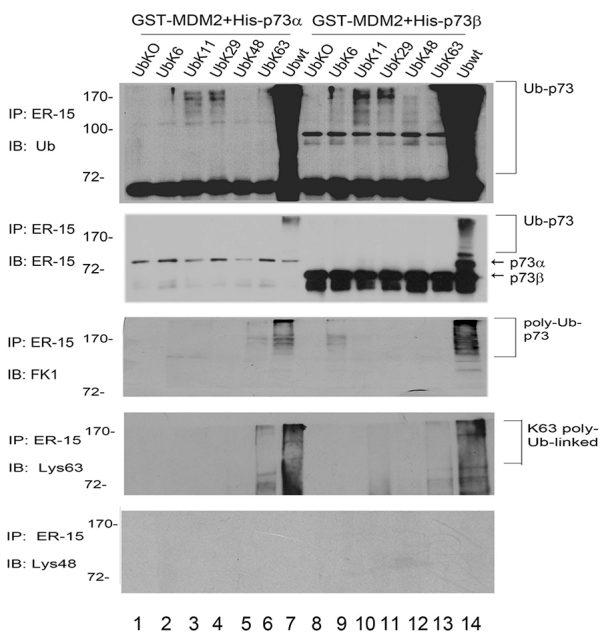

$\mathrm{F}$

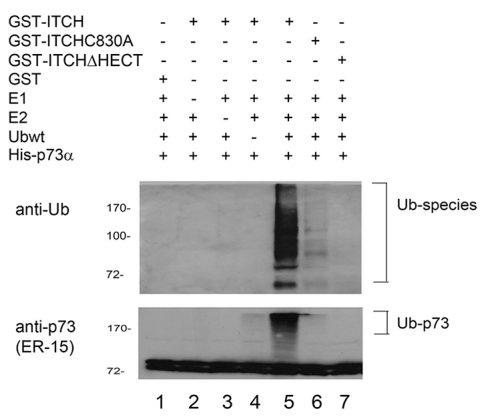

Figure 3: MDM2 is an E3 ligase for p73 in vitro. A. and B. GST-MDM2 was evaluated for its capacity to ubiquitinate purified His-p73 $\alpha$ and His-p73 $\beta$ using immunoblotting with anti-Ub to reveal ubiquitinated products (upper image) and an antibody directed to p73 (ER-15) to reveal ubiquitinated p73 species. C. MDM2 preferentially utilizes Ub with Lys residues at Lys11 and Lys29 for p73 ubiquitination in vitro. Affinity-purified GST-MDM2, His-p73 $\alpha$, or His-p73 $\beta$ was added to bacterial extracts containing recombinant E1 and E2 (UbcH5b), Ubwt or ubiquitin mutants. Immunoblotting was performed with anti-Ub to reveal ubiquitinated products (upper image) and anti-p73 (ER-15) to reveal ubiquitinated p73 species (lower image). D. As in (C) but after the ubiquitination reaction, the samples were immunoprecipitated with RIPA buffer and anti-p73 (ER-15) antibody and analyzed by immunoblotting with anti-Ub (upper image), anti-p73 (ER-15, middle image), polyubiquitin-specific FK-1 (third image), and anti-ubiquitin, Lys63-specific and Lys48-specific antibodies (lower image) as indicated. E. Western blot analysis of p $73 \alpha$ or p $73 \beta$ ubiquitination was performed with a p73-specific antibody (ER-15, upper image), a polyubiquitinspecific antibody FK-1(lower image), and varying amounts of GST-MDM2 (0.1X: 0.3 ng; 1X: 3 ng; 10X: $30 \mathrm{ng}$ ) in the presence of Ubwt or UbKO. E1 and E2 were included in all reactions. Asterisk (*) indicates the migration position of p73-Ub conjugates. F. GST-ITCH, GST-ITCHC830A and GST-ITCH $\triangle$ HECT were evaluated for E3 activity in the presence of recombinant $\mathrm{E} 1, \mathrm{E} 2$ (UbcH5b), ubiquitin and $\mathrm{p} 73 \alpha$ protein. Following the ubiquitination reaction, the samples were analyzed by western blotting with ubiquitin-specific and p73-specific (ER-15) antibodies. 
A

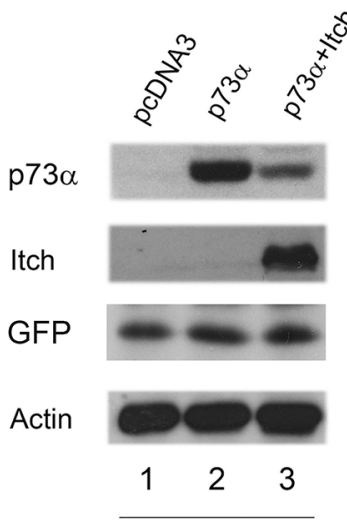

Mdm2-null MEFs
B

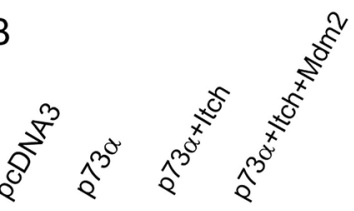

$p 73 \alpha$

Itch

Mdm2

GFP

Actin

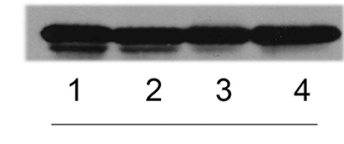

C

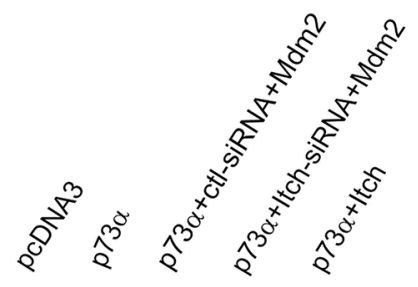

p73 $\alpha$

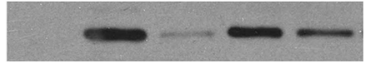

Itch

Mdm2

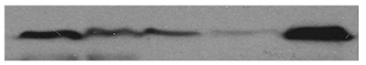

GFP

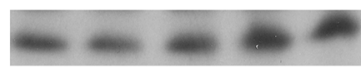

Actin

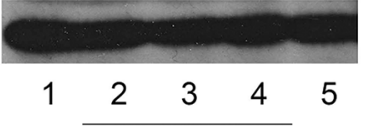

Mdm2-null MEFs
D

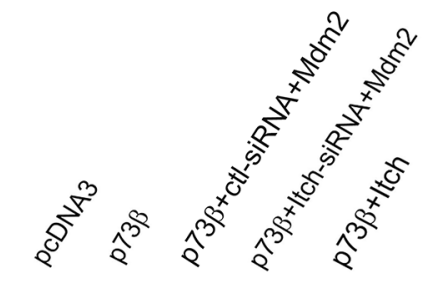

p73 $\beta$

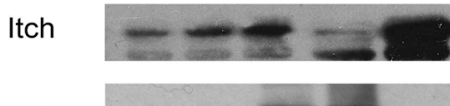

Mdm2

GFP

Actin
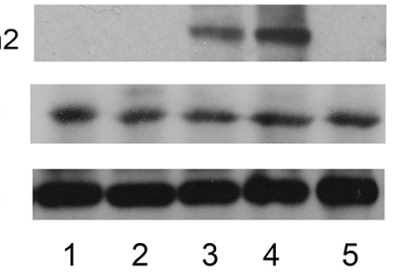

E

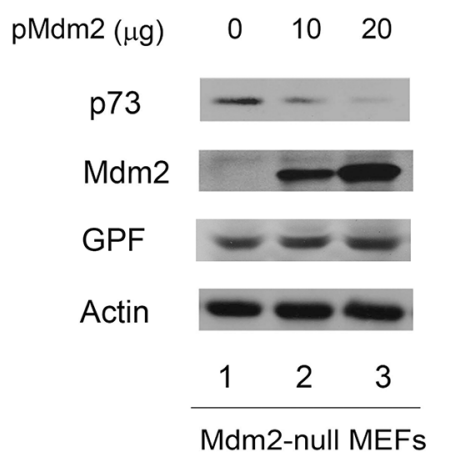

Figure 4: Overexpression of Mdm2 promotes p73 degradation in Mdm2-null MEFs. A. Mdm2 null MEFs were transfected with plasmids expressing p $73 \alpha$ or an empty vector with Myc-tagged Itch, along with a GFP expressing plasmid (pEGFP) and analyzed by western blotting with a p73-specific antibody (H-79), a Myc-specific antibody for Myc-Itch, a GFP-specific antibody (B-2), and actin as a loading control. B. The same procedure as (A) was used, except that Mdm2 null MEFs were cotransfected with plasmids expressing GFP, p73 $\alpha$ along with Itch and Mdm2. C. and D. Mdm2-null MEFs were transfected with an Itch-specific siRNA or a control siRNA. Thirty hours later, the cells were transfected with plasmids expressing GFP, Mdm2 and analyzed by western blot using anti-p73, anti-Itch, anti-p53 (Pab421), anti-GFP (B-2), and anti-Mdm2 (MD-219) antibodies as indicated. E. Mdm2-null MEFs were transfected with plasmids expressing GFP and increased amounts of Mdm2 and analyzed by western blotting with p73-specific (H-79), GFP-specific (B-2) and Mdm2-specific (MD-219) antibodies. 


\section{Mdm2 interacts with Itch and regulates the stability of $\mathbf{p} 73$ proteins}

MDM2 was recently reported to promote p73 degradation through interaction with ITCH in HeLa cells [27]. We were able to detect weak interaction between Itch and transfected Mdm2 in wild type MEFs compared to that of Mdm2 null MEFs in the presence of the proteasome inhibitor MG132 (Figure 5A). To further determine if $\mathrm{Mdm} 2$ regulates p73 stability, wild type MEFs and Mdm2 null MEFs were treated with cycloheximide to inhibit de novo protein synthesis. We observed that the halflife of endogenous p73 was approximately $1 \mathrm{hr}$ in wild type MEFs. By contrast, the half-life of p73 increased to approximately $3 \mathrm{hr}$ in Mdm2 null MEFs (Figure 5B, 5C). These data reveal that $\mathrm{Mdm} 2$ is able to regulate the stability of p73 in Mdm2 null MEFs. In the co-IP experiment, immunoprecipitated p73 was heavily ubiquitinated in the presence of Itch (Figure 5D). The data indicates that Itch functions independently of Mdm2. Furthermore, we investigated whether MDM2 mediates p73 ubiquitination through ITCH in different cell types. Endogenous ITCH was subjected to ablation by ITCH-siRNA in human HEK293 cells. Two days later, cells were transfected with MDM2 and HA-tagged ubiquitin (HA-Ub) expression plasmids. Extracts were immunoprecipitated with a p73specific antibody (ER-15) and analyzed by western blotting with an HA-specific antibody to detect ubiquitinated p 73 (Figure 5E, upper image) and a p73-specific antibody (ER-15) to detect all p73 (Figure 5E, lower image). Heavily ubiquitinated $\mathrm{p} 73$ (or proteins that coimmunoprecipitated with p73) was detected when the ITCH-siRNA treated cells transfected with MDM2 compared with cells transfected with an empty vector or control-siRNA (Figure 5E). The p73 immunoblot also reveal ubiquitination of p73 when cells were transfected with MDM2; however, the levels of p73 were not significantly changed in HEK 293 cells (Figure 5E, lower image). These data indicate that MDM2 promotes p73 ubiquitination independently of ITCH. We further tested the ability of MDM2 to regulate the level of p73 in HEK293 cells. Notably, the half-life of p73 remained unchanged in the presence of MDM2 compared with pcDNA3 (Supplementary Figure S2A). By contrast, the half-life of $\mathrm{p} 73$ was greatly decreased in the presence of ITCH. We also observed that the halflife of endogenous p73 in the presence of control-siRNA or MDM2-siRNA was approximately $4 \mathrm{hr}$. The halflife increased to approximately $6 \mathrm{hr}$ in cells depleted of ITCH (Supplementary Figure S2B). These data revealed that MDM2 is unable to regulate the stability of p73 in HEK293 cells. Moreover, we confirmed that p73 $\alpha$ was heavily ubiquitinated in the presence of Mdm2 or Itch and $\mathrm{p} 73 \beta$ was a lesser extent in the presence of Mdm2 or Itch in Mdm2 null MEFs using His ubiquitin pull down assay (Figure 5F).

\section{MDM2 represses p73 apoptosis and cell cycle arrest but not $\mathbf{p} 73$-dependent transcriptional activity via $\mathrm{E} 3$ ligase activity in tested cells}

To determine the functional consequences of the interaction of MDM2 with p73, we examined the effect of MDM2 or ITCH expression on p73mediated transcriptional activity. H1299 cells were cotransfected with a luciferase reporter construct (p21-luc) containing the $\mathrm{p} 53$ binding site from the $\mathrm{p} 21^{\mathrm{WAF} 1}$ promoter and $\mathrm{p} 73$ alone or with MDM2 or ITCH. Both MDM2 and ITCH repress p73mediated transactivation activity (Figure 6A), consistent with previous studies $[9,18,19]$. Notably, the MDM2 $\triangle$ RING mutant significantly repressed p73dependent transactivation, suggesting that MDM2 impairs the transactivation function of $\mathrm{p} 73$ without targeting it for degradation and indicating that the RING domain of MDM2 is not necessary for MDM2 inhibition of p73 transcriptional activity. A portion of cell extracts were lysed and analyzed by western blots (Figure 6B). To further assess the involvement of endogenous MDM2 in the regulation of p73 ubiquitination, HEK293 cells were pretreated with control-siRNA, MDM2-siRNA, ITCH-siRNA, or MDM2-siRNA and ITCH-siRNA. The cells were also transfected with the $\mathrm{p} 21^{\mathrm{WAF}}$-luc reporter. As shown in Figure $6 \mathrm{C}$, the activity of $\mathrm{p} 21^{\mathrm{WAF}}$-luc was increased in cells depleted of MDM2 or ITCH by siRNA. The transcriptional activity of $\mathrm{p} 73$ was further increased in cells depleted of both MDM2 and ITCH (Figure 6C). This is consistent with the observation that $\mathrm{p} 73$ transcriptional activity is inhibited by MDM2 overexpression (Figure 6A). The mechanism of MDM2-mediated inhibition of p73 transactivation activity involves MDM2 binding to the N-terminal domain of p73, which blocks the ability of p73 to activate target gene transcription. Next, we examined whether overexpression of MDM2 inhibits p73-dependent apoptosis. Transient expression experiments followed by FACS analysis (using annexin $\mathrm{V}$ staining) were performed to determine whether MDM2 expression can rescue cells from p73-dependent cell death. As shown in Figure 6D, expression of p73 alone resulted in apoptosis. However, apoptosis is largely prevented by coexpression of MDM2 [19]. The MDM2 $\triangle$ RING mutant was unable to rescue cells from p73-dependent cell death, which suggests that the E3 ligase activity of MDM2 is required to inhibit p73-mediated apoptosis. The acidic domain (AD) of MDM2 has been reported to play a critical role in p53 ubiquitination [28, 29]. Neither the RING nor the AD domain of MDM2 alone is sufficient for efficient p53 ubiquitination [28, 29]. However, we observed that the MDM2 $\Delta 222-303$ mutant (deletion of $\mathrm{AD}$ of MDM2) was able to partially prevent p73-dependent apoptosis, indicating that the AD domain of MDM2 is not absolutely necessary for the repression of 


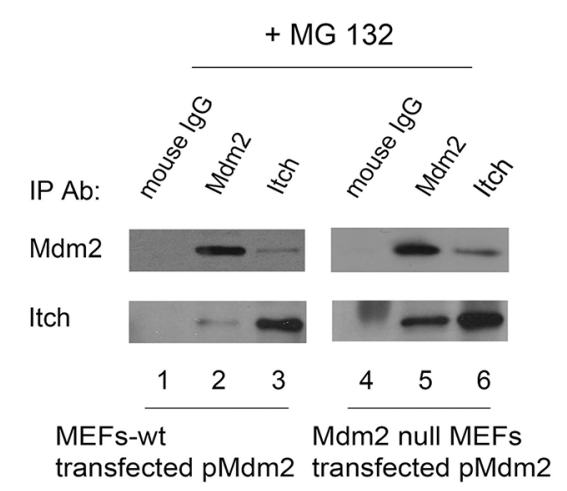

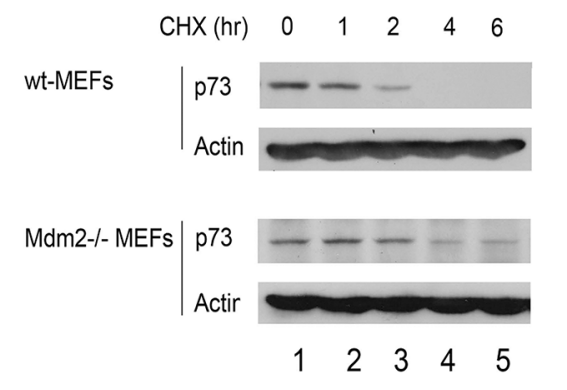

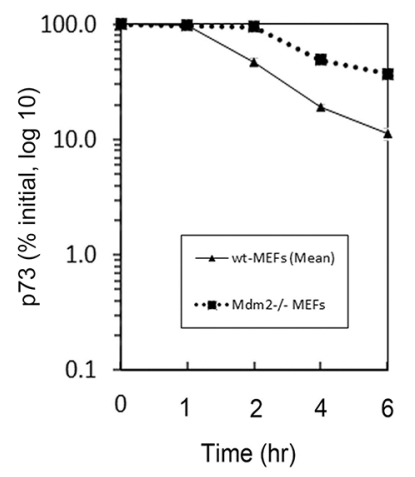

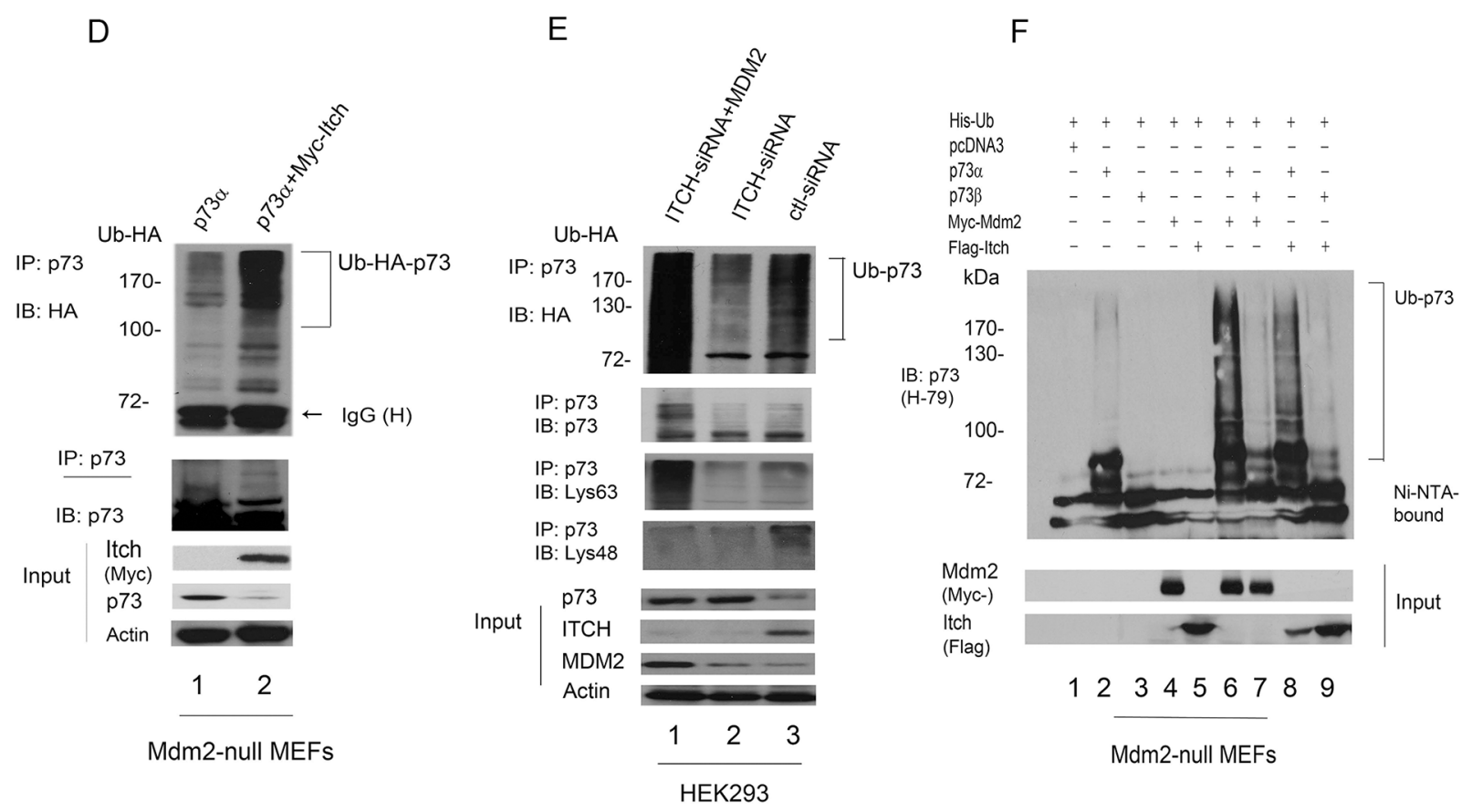

Figure 5: Mdm2 interacts with Itch and regulates the stability of p73 in Mdm2-null MEFs. A. Mdm2 interacts with Itch in vivo. Wild type MEFs and Mdm2-null MEFs were transfected with a plasmid expressing Mdm2. The cells were treated with the proteasome inhibitor MG132 $(20 \mu \mathrm{M})$ for 6 hours prior to harvest. The cell extracts were immunoprecipitated with anti-Mdm2 or anti-Itch antibodies and analyzed by western blot with indicated antibodies. B. Wild type MEFs and Mdm2 null MEFs cells were treated with cycloheximide (CHX) (20 $\mu \mathrm{g} / \mathrm{ml})$ as indicated. Endogenous p73 levels were determined by immunoblotting with a p73-specific antibody (H-79). An antibody against $\beta$-actin was used as a loading control. C. Expression levels were determined by densitometry of the immunoblots in (B) Errors bars indicate the SEM $(n=3)$. D. Mdm2 null MEFs were transfected with plasmids expressing HA-Ub and p73 $\alpha$ or Myc-Itch. Cell extracts were immunoprecipitated with a p73-specific antibody (H-79) and analyzed by western blotting with HA-specific, Myc-specific (for Itch), and p73-specific antibodies. E. HEK293 cells were transfected with ITCH-siRNA or control-siRNA. Two days later, the cells were further transfected with plasmids expressing HA-Ub and an MDM2 expression plasmid or empty vector as indicated. Cell extracts were immunoprecipitated with a p73-specific antibody (ER-15) and analyzed by western blotting with HA-specific, p73-specific, Lys63-specific and Lys48-specific antibodies (lower image) as indicated. Direct western blots for p73, ITCH, MDM2 and actin are shown in the lower panel. F. Mdm2 null MEFs were transfected with plasmids expressing His-Ub, p $73 \alpha$ or p $73 \beta$, and Mdm2 or Itch. His-ubiquitinated proteins were isolated from denatured whole extract extracts, and analyzed by western blot with a p73 specific antibody (H-79). Direct western blots for Mdm2 and Itch are shown in the lower panels. 
p73dependent apoptosis (Figure 6D). This indicates that MDM2-mediated p73 ubiquitination is linked to inhibition of the apoptotic function of $\mathrm{p} 73$. To further determine whether endogenous MDM2 is required to inhibit p73dependent apoptosis, H1299 cells were exposed to either control-siRNA or MDM2-siRNA. Two days later, these cells were transfected with $\mathrm{p} 53, \mathrm{p} 73 \alpha$, or $\mathrm{p} 73 \beta$ and analyzed by flow cytometry using annexin V staining. Our findings revealed that depletion of MDM2 resulted in additional apoptosis when cells were transfected with $\mathrm{p} 53$, $\mathrm{p} 73 \alpha$, or p $73 \beta$, confirming that MDM2 plays an important role in regulating p73 apoptosis (Figure 6E).

Cell cycle arrest mediated by p73 is an important tumor suppression function of $\mathrm{p} 73$. To determine whether MDM2 can inhibit p73-induced G1 arrest, human bladder carcinoma EJ cells, which lack functional p53 [30, 31], were transfected with a plasmid expressing p $73 \alpha$ alone, with MDM2, or with MDM2 2 RING. Cell cycle analysis by propidium iodide staining and flow cytometry was performed. The relative proportion of cells in each phase (G1, S and G2/M) of the cell cycle was determined using the automated ModFit program (Verity Software House Incorporated). It is important to compare the $\mathrm{G} 1 / \mathrm{S}$ ratios; an increase in the $\mathrm{G} 1 / \mathrm{S}$ ratio has been used as an indicator of G1 arrest [20-22]. Overexpression of p73 $\alpha$ exhibited an increase in the proportion of cells in G1 and a decrease in the proportion of cells in S phase, resulting in an increase in the G1/S ratio from 1.11 to 4.49 (Figure 6F). We observed that p73-induced G1 arrest was partially prevented by overexpression of MDM2; however, MDM2 $\triangle$ RING was unable to prevent $\mathrm{p} 73$-induced G1 arrest (Figure 6F), suggesting that the E3 ligase activity of MDM2 is required to inhibit p73-dependent cell cycle arrest. These data demonstrate that the E3 ligase activity of MDM2 is involved in the regulation of p73-dependent apoptosis and checkpoint control.

\section{DISCUSSION}

The protein p73 was isolated as a p53 homolog and exhibits similar functions, such as transactivation of p53 target genes, induction of apoptosis, and growth suppression. MDM2 has been reported to bind to p73 and inhibits p73dependent transactivation and apoptosis. While MDM2 regulates p53 stability, it is not involved in the regulation of $p 73$ protein stability $[18,19]$. It has been reported that Nutlin-3, a small molecule inhibitor of MDM2, can disrupt endogenous p73-MDM2 binding and induce apoptosis in the absence of p53 [32]. Busuttil et al observed that NF- $\mathrm{\kappa B}$ induced Mdm2 expression in activated $\mathrm{T}$ cells [33]. Mdm2 prevents Bim-mediated apoptosis via binds and inhibition of p73 [33]. However, the molecular mechanism by which MDM2 represses p73 function is not clear. In this study, we elucidated the mechanism by which MDM2 negatively regulates p73 function. This is the first report in which p73 ubiquitination mediated by MDM2 has been linked to MDM2 repression of p73-dependent apoptosis and cell cycle arrest but not for p73-dependent transcriptional activity.

To investigate the role of MDM2 in a physiological setting, endogenous MDM2 was depleted by siRNA. We demonstrated that p73 ubiquitination was greatly decreased in cells depleted of MDM2, suggesting that MDM2 is required for $\mathrm{p} 73$ ubiquitination in vivo. We also observed that overexpression of MDM2 promoted p73 ubiquitination. By contrast, MDM2 $\triangle$ RING was unable to mediate p73 ubiquitination, indicating that the E3 ligase activity of MDM2 is required for p73 ubiquitination. Intriguingly, MDM2 utilizes multiple Ub chains (Lys11, Lys29, and Lys63) to mediate $\mathrm{p} 73$ ubiquitination in vivo and in vitro. It has been reported that Lys11-, Lys 29-, and Lys48 linked chains are targeted for degradation by the $26 \mathrm{~S}$ proteasome $[34,35]$. Lys63-linked chains appear to be used primarily for nonproteasomedependent regulation of processes such as DNA repair, endocytosis, and chromatin remodeling $[7,36]$. The biological importance of K48linked polyubiquitinated chains is confirmed by the lethality of the K48R mutation in the yeast Saccharomyces cerevisiae [37]. Monoubiquitination is involved in a variety of cellular functions, including transcriptional activation, protein-protein interactions, and membrane trafficking [38]. Our findings revealed that high level of MDM2 mediates polyubiquitination of $\mathrm{p} 73$. We detected p73 polyubiquitination in the presence of Ubwt and weak p73 polyubiquitination in the presence of Lys63 or Lys6 chains. This may explain why MDM2 is unable to degrade p73 proteins in certain cell types. A recent study proposed that degradation of some polyubiquitinated proteins requires two binding interactions, including polyubiquitin chains and an intrinsic proteasomal binding element in the substrates [26]. It is also possible that the proteasomal binding element in p73 is inactive. Additional studies are required to address this molecular mechanism.

MDM2 did not promote p73 degradation in most tested cell lines. Recently, Kubo et al. reported that MDM2 mediates $\mathrm{p} 73$ degradation through interaction with Itch in HeLa cells [27]. Our finding that MDM2 has an undetectable effect on $\mathrm{p} 73$ at the protein level in certain cell types is in agreement with previous studies. We were unable to detect an MDM2 interaction with ITCH in H1299 and HEK293 cells, also consistent with previous reports [27]. We show that Itch (the mouse homolog of ITCH) degrades p73 independently of Mdm2 in Mdm2 null MEFs. Notably, overexpression of Mdm2 promotes p73 degradation in Mdm2-null MEFs. We demonstrate that Itch is essential for overexpression of Mdm2 mediated p73 degradation in Mdm2-null MEFs. Moreover, we observe that the E3 ligase activity of MDM2 is required for MDM2 to suppress p73-dependent apoptosis and cell cycle arrest. Our findings provide evidence for a link between the E3 ligase activity of MDM2 and its role in repressing $\mathrm{p} 73$ function. 
A

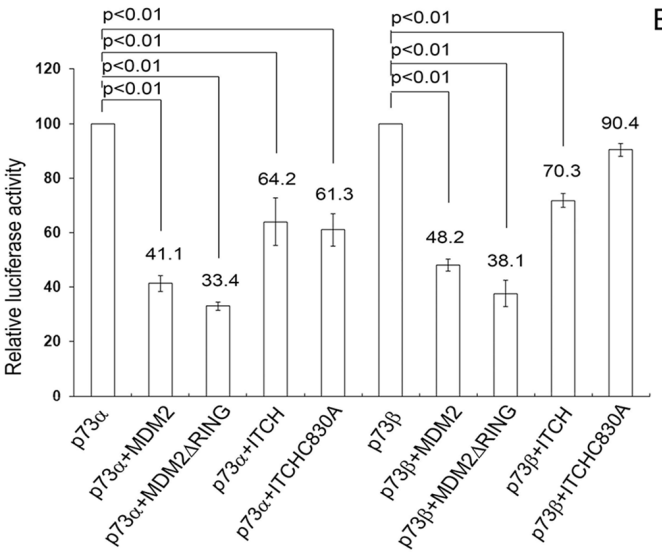

B
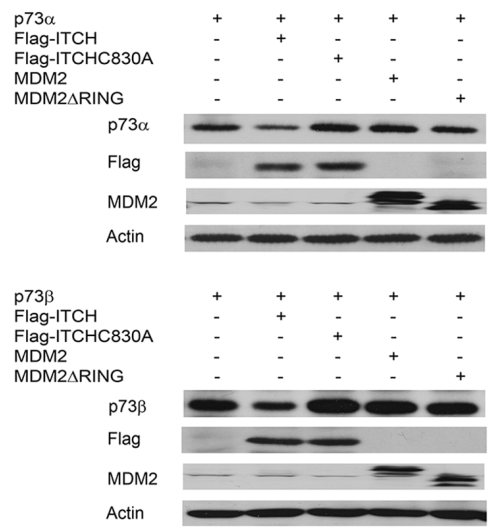

C
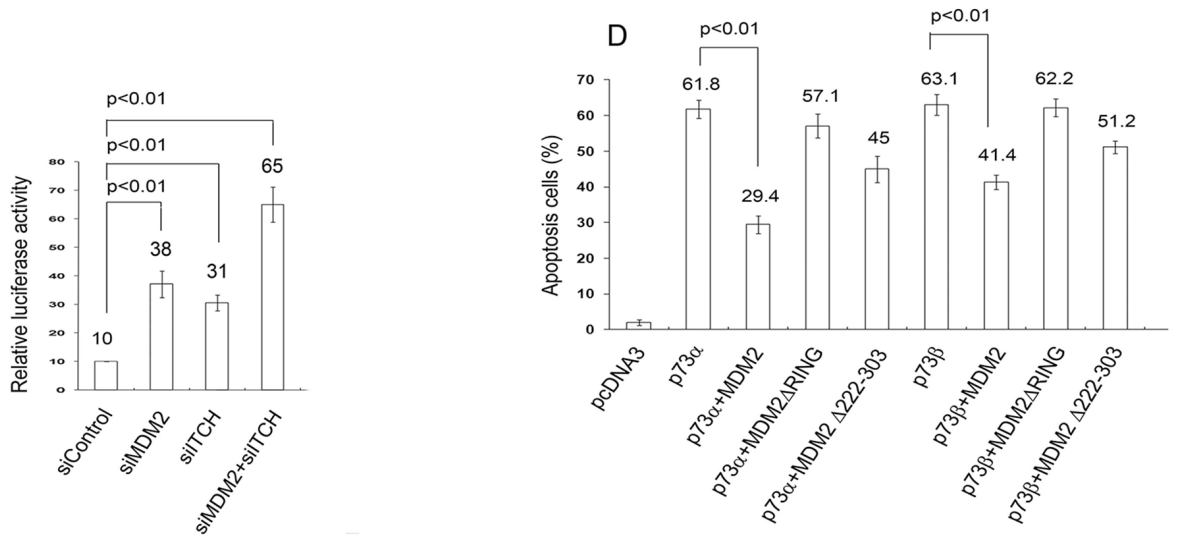

E
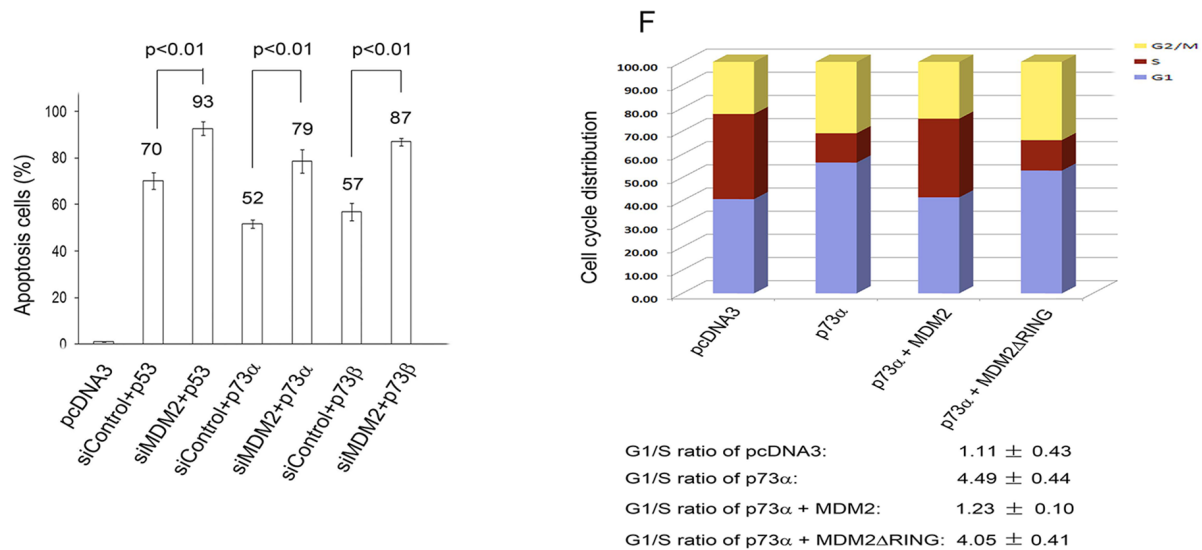

Figure 6: MDM2 inhibits p73-dependent apoptosis and cell cycle arrest but not p73-dependent transcriptional activity. A. H1299 cells were cotransfected with a p21-Luc reporter plasmid and the p $73 \alpha$ or p $73 \beta$ expression construct in combination with the MDM2, MDM2 $\triangle$ RING, ITCH, or ITCH C830A expression constructs or empty vector (pcDNA3.1). The transcriptional activity of $\mathrm{p} 73$ is shown, and error bars indicate the $\operatorname{SEM}(n=3) . p<0.01$ (2-tailed Student t test). B. Western blots of p73 $\alpha, \mathrm{p} 73 \beta$, ITCH, ITCHC830A, MDM2 and MDM2 2 RING with p73-specific (ER15), Flag-specific for ITCH and ITCHC830A and MDM2-specific antibodies were indicated. C. HEK293 cells were transfected with MDM2-siRNA (siMDM2), ITCH-siRNA (siITCH), control-siRNA, or MDM2-siRNA and ITCH-siRNA. Two day later, the cells were transfected with a p21 Luc reporter plasmid and a p $73 \alpha$ expression plasmid. The transcriptional activity of $\mathrm{p} 73$ is shown, and error bars indicate the $\operatorname{SEM}(n=3) \cdot p<0.01$ (2-tailed Student $t$ test). D. H1299 cells were cotransfected with a CD20 expression construct, pcDNA3-p73 $\alpha$, or p73 $\beta$ (3 $\mu \mathrm{g})$ and pcDNA3-MDM2 (15 $\mu \mathrm{g})$, pcDNA3-MDM2 $\Delta$ RING $(15 \mu \mathrm{g})$, or pcDNA3-MDM2 $\Delta 222-303$ as indicated. The inhibitory effect of MDM2 on p73-dependent apoptosis was determined by annexin V staining of CD20-positive cells and flow cytometry. Error bars indicate the SEM. $(n=3) . p<0.01$ (2-tailed Student $t$ test). E. H1299 cells were transfected with MDM2-siRNA or control-siRNA for $30 \mathrm{~h}$. The cells were then transfected with the p53, p73 $\alpha$, or p73 $\beta$ expression constructs and CD20 expression plasmids. The number of surviving CD20-positive cells was measured by flow cytometry $24 \mathrm{hr}$ after transfection. $p<0.01$ (2-tailed Student $t$ test). F. Human bladder carcinoma EJ cells were transfected with p73 $\alpha$ alone or with MDM2, MDM2 2 RING, or empty vector (pcDNA3). The cell cycle profile was determined by propidium iodide staining and flow cytometry. The results represent the average of triplicate experiments. 
An important question raised by our results is whether the stability of p73 is regulated by MDM2 through distinct mechanisms. First, MDM2 interacts with ITCH in certain cell types, which suggests that additional co-factor(s) are required for the interaction. No evidence showed that MDM2 directly bound to ITCH in vitro. Second, the complex formation between MDM2 and ITCH may not be stable in some cell types. That is partially explained why MDM2 repressed p73-dependent apoptosis in some cell types, which we do not see significant p73 degradation. Third, it is possible that additional co-factor(s) inhibit the interaction between MDM2 and ITCH in some cell types. However, this inhibition is removed/or turned off in certain cell types (such as HeLa and Mdm2-null MEFs). Mdm2 and Itch are E3 ubiquitin ligases that regulate p73 ubiquitination and degradation. Both the RING domain and the HECT domain share similar surface structures in E2 binding, but use distinct mechanisms for substrate ubiquitination. It will be interesting to further address the effect of Mdm2 on Itch mediated p73 regulation and function.

Controlling p73 stability is critical to understanding the regulation of the protein activity in the cell. In this study, we elucidated the molecular mechanism by which MDM2 inhibits p73 function. Our findings also demonstrated that MDM2 is a critical negative regulator of $\mathrm{p} 73$.

\section{MATERIALS AND METHODS}

\section{Plasmids and antibodies}

GST-MDM2, p21-Luc, pCMV-Bam-MDM2, and MDM2 $\Delta$ RING have been described previously [20, 21]. Myc-Mdm2 was kindly provided by Dr. Jochemsen. All $\mathrm{Ub}$ and $\mathrm{Ub}$ mutants were PCR-amplified and subcloned into pET28a. The mouse Mdm2 was also cloned into pcDNA3 and confirmed by sequence. Flag-p $73 \alpha$, p $73 \beta$, and p73 mutants were generated by PCR and subcloned into pCMV-Tag1 (Stratagene). p73 $\alpha$ and p $73 \beta$ were also cloned into pcDNA3 without tag. All PCR products were confirmed by sequencing. Anti-p73 (Ab-1 and Ab3, Oncogene Science; H-79, Santa Cruz Biotechnology; ab40658, Abcam), anti-MDM2 (2A10, Calbiochem; SMP14, Santa Cruz Biotechnology; MD-219, Sigma), anti-Myc (9E10, Roche), anti-Flag (M5, Sigma), anti-GFP (B-2, Santa Cruz Biotechnology), anti-GST (B-14, Santa Cruz Biotechnology), anti-HA (12CA5, Roche), antiubiquitin (BD Bioscience), anti-actin (Sigma), anti-CD20 (Pharmingen), and polyubiquitin-specific FK-1, antiubiquitin, Lys63-specific and Lys48-specific antibodies (Millipore) were used according to the manufacturers' instructions.

\section{Cell culture and DNA transfection}

All cells were maintained in Dulbecco's modified Eagle's medium (DMEM) supplemented with 10\% fetal bovine serum. The $\mathrm{p} 53^{-/-} \mathrm{Mdm} 2^{-/}$double null MEFs were kindly provided by Dr. G. Lozano. H1299 cells and Saos2 cells were transfected using the calcium phosphate method. Mdm2 null MEFs were transfected using Lipofectamine 2000 (Invitrogen).

\section{siRNA experiments}

For siRNA experiments, HEK293 cells were transfected with siRNA using Lipofectamine 2000 (Invitrogen). The Itch target sequences were AAGTGCTTCTCAGAATGATGA and AACCACAA CACACGAATTACA, and the scrambled sequence was AATTCTCCGAACGTGTCACGT [as described previously (9)]. pSuper.neo.gfp-Mdm2 siRNA (GACAAAGAAGAGAGTGTGG) was a kind gift from Dr. C. Blattner.

\section{Expression and recombinant protein preparation}

All GST or His-tagged recombinant proteins were expressed in E. coli BL21 (DE3) (Novagen), treated with isopropyl- $\beta$-D-thiogalactoside to induce fusion protein expression, and purified using glutathione Sepharose 4B (Amersham) for GST-fusion proteins or $\mathrm{Ni}^{2}$-NTA agarose (Qiagen) for His-fusion proteins.

\section{Immunoprecipitation and measurement of p73 half-life}

Cells were lysed in $50 \mathrm{mM}$ Tris- $\mathrm{HCl}(\mathrm{pH} \mathrm{8.0)}$, $5 \mathrm{mM}$ EDTA, $150 \mathrm{mM} \mathrm{NaCl}, 0.5 \% \mathrm{NP}-40$ containing a protease inhibitor tablet (Roche) and immunoprecipitated with specific antibodies. The immune complexes were collected with protein A agarose beads and washed 4 times with lysis buffer. The immunoprecipitates were analyzed by SDS PAGE followed by autoradiography. To measure the p73 half-life, wild type MEFs and Mdm2 null MEFs were treated with $25 \mu \mathrm{g} / \mathrm{ml}$ cycloheximide (CHX) to inhibit de novo protein synthesis. Protein levels were monitored by immunoblotting with a p73 specific antibody (H-79) at the indicated time points. The relative amount of $\mathrm{p} 73$ protein was determined by densitometry and normalization using $\beta$-actin.

\section{In vitro ubiquitination assay}

The in vitro ubiquitination assay was performed as described previously [20-22] with some modifications. For MDM2-mediated ubiquitination, rabbit E1 (20-40 ng, Calbiochem), UbcH5b (100 ng, Calbiochem), ubiquitin or His-ubiquitin (5 $\mu \mathrm{g}$, Sigma), His-p73, and GST-MDM2 (0.2-0.5 $\mu \mathrm{g})$ were added to ubiquitination buffer $(50 \mathrm{mM}$ Tris- $\mathrm{HCl}, \mathrm{pH} 7.4,2 \mathrm{mM}$ ATP, $5 \mathrm{mM} \mathrm{MgCl}$, and $2 \mathrm{mM}$ DTT) to obtain a final volume of $30 \mu \mathrm{l}$. The reactions were incubated at $30^{\circ} \mathrm{C}$ for $1.0-1.5 \mathrm{hr}$. The reactions were stopped with $2 \mathrm{x}$ SDS loading buffer, 
resolved on SDS-PAGE gels, and analyzed by western blotting.

\section{In vivo ubiquitination assay}

Cells were transfected with expression plasmids encoding p73, MDM2, or Itch alone or in combination with HA-tagged ubiquitin, HA-ubiquitin mutants, MycUbwt, or a Myc-Ub4KR mutant. After $30 \mathrm{hr}$, cells were harvested, lysed with RIPA buffer [50mM Tris-HCl $\mathrm{pH} 7.6,150 \mathrm{mM} \mathrm{NaCl}, 1 \% \mathrm{NP}-40,1 \%$ sodium deoxycholate, $0.1 \%$ SDS, and protease inhibitors (Roche)], and immunoprecipitated with the indicated antibodies. Immune complexes recovered with protein A-Sepharose were washed 4 times with RIPA buffer, separated by $10 \%$ SDS-PAGE, and analyzed by IP/immunoblotting as described previously [20-22].

\section{His-ubiquitin pull-down assay}

Cells were transfected with His-tagged ubiquitin and indicated expression plasmids. Thirty hours after transfection, cells were resuspended in Buffer A $(6 \mathrm{M}$ guanidine- $\mathrm{HCl}, 0.1 \mathrm{M} \mathrm{Na} 2 \mathrm{HPO} 4 / \mathrm{NaH} 2 \mathrm{PO} 4,10 \mathrm{mM}$ Tris$\mathrm{HCl}, \mathrm{pH}$ 8.0, $10 \mathrm{mM}$ imidazole at $\mathrm{pH}$ 8.0) and sonicated. Cell lysates were added to $50 \mu \mathrm{l}$ of equilibrated Ni-NTA agarose and were allowed to incubate for $3 \mathrm{~h}$ at room temperature. Beads were then washed one time with Buffer A, followed by two washes with Buffer A/TI (1 vol of Buffer A, 3 vol of Buffer TI [25 mM Tris-Cl, $20 \mathrm{mM}$ imidazole at $\mathrm{pH}$ 6.8]), and one wash with Buffer TI; all washes were $1 \mathrm{ml}$. After extensively washing, the precipitates were boiled with SDS loading buffer and then subjected to SDS-PAGE followed by immuno-blot analysis.

\section{Cell cycle analysis}

(described previously [20-22]).

\section{Apoptosis assay}

(described previously [20-22]).

\section{Luciferase assay}

As described previously [20-22], pGL3-E1bTATA contains a minimal promoter consisting of a TATA box downstream of one copy of the p53 binding site from the 5 'end of the p21WAF promoter, referred to as $\mathrm{p} 21$-Luc. A $\beta$ galactosidase reporter construct, $\mathrm{pCMV}-\beta$-gal (Promega), was included in all the transfection mixtures. Luciferase activity was measured 2 days posttransfection on samples containing equivalent amounts of protein using an LB9507 luminometer and the luciferase assay reagent (Promega). All values were normalized to $\beta$-galactosidase activity.

\section{ACKNOWLEDGMENTS}

We gratefully acknowledge Drs. W. Gu (Columbia University) for the His-Ubiquitin (wt) and His-Ubiquitin KO plasmids, A. Marchese (Loyola University Chicago) for GST-ITCH and GST-ITCHC830A, A.G. Jochemsen (Erasmus University Medical Center) for the Myc-Mdm2 plasmid, Y. Yarden (Weizmann Institute of Science) for Myc-wtUb and Myc-4KRUb plasmids, T. Pawson (Samuel Lunenfeld Research Institute) for Myc-ITCH and MycITCH mutant (C830A), C. H. Arrowsmith (Ontario Cancer Institute) for pET15b-His-p73 $\alpha$ and pET15b-His-p73 $\beta$, C. Blattner (Universität Heidelberg) for pSuper.neo.gpfMdm2 siRNA plasmid, and G. Lozano (University of Texas, M.D. Anderson Cancer Center) for Mdm $2^{--T r p} 53^{-/-}$ MEFs as described in the text.

\section{FUNDING}

This work was supported by grants from Women \& Children's Health Research Institute (WCHRI), the Alberta Heritage Foundation for Medical Research (AHFMR), Breast Cancer Foundation, and Canadian Institutes of Health Research (CIHR) to R.P.L.

\section{CONFLICTS OF INTEREST}

The authors declare no conflict of interest.

\section{REFERENCES}

1. Yang A, Walker N, Bronson R, Kaghad M, Oosterwegel M, Bonnin J, Vagner C, Bonnet H, Dikkes P, Sharpe A, McKeon F, Caput D. p73-deficient mice have neurological, pheromonal and inflammatory defects but lack spontaneous tumours. Nature. 2000; 404:99-103.

2. Yang A, McKeon F. p63 and p73: p53 mimics, menace and more. Nature Rev Mol. Cell Biol. 2000; 1:199-207.

3. Levrero M, De Laurenzi V, Costanzo A, Gong J, Wang JY, Melino G. The p53/p63/p73 family of transcription factors: overlapping and distinct functions. J Cell Sci. 2000; 113:1661-1670.

4. Jacobs WB, Kaplan DR, Miller FD. The p53 family in nervous system development and disease. J of Neurochemistry. 2006; 97:1571-1584.

5. Ahomadegbe JC, Tourpin S, Kaghad M, Zelek L, Vayssade M, Mathieu MC, Rochard F, Spielmann M, Tursz T, Caput D, Riou G, Bénard J. Loss of heterozygosity, allele silencing and decreased expression of $\mathrm{p} 73$ gene in breast cancers: Prevalence of alterations in inflammatory breast cancers. Oncogene. 2000; 19:5413-5418.

6. Puig P, Capodieci P, Drobnjak M, Verbel D, Prives C, Cordon-Cardo C, Di Como CJ. p73 expression in human normal and tumor tissues: loss of $\mathrm{p} 73 \alpha$ expression is associated with tumor progression in bladder cancer. Clin Cancer Res. 2003; 9:5642-5651. 
7. Park HR, Kim YW, Park JH, Maeng YH, Nojima T, Hashimoto H, Park YK. Low expression of p63 and p73 in osteosarcoma. Tumori. 2004; 90:239-243.

8. Flores ER, Sengupta S, Miller JB, Newman JJ, Bronson R, Crowley D, Yang A, McKeon F, Jacks T. Tumor predisposition in mice mutant for p63 and p73: Evidence for broader tumor suppressor functions for the p53 family. Cancer Cell. 2005; 7:363-372.

9. Rossi M, De Laurenzi V, Munarriz E, Green DR, Liu YC, Vousden KH, Cesareni G, Melino G. The ubiquitin-protein ligase Itch regulates p73 stability. EMBO J. 2005; 24:836-848.

10. Fakharzadeh SS, Trusko SP, George DL. Tumorigenic potential associated with enhanced expression of a gene that is amplified in a mouse tumor cell line. EMBO J. 1991; 10:1565-1569.

11. Oliner JD, Pietenpol JA, Thiagalingam S, Gyuris J, Kinzler KW, Vogelstein B. Oncoprotein MDM2 conceals the activation domain of tumor suppressor p53. Nature. 1993; 362:857-860.

12. Leng P, Brown DR, Deb S, Deb SP. N-terminal 130 amino acids of MDM2 are sufficient to inhibit p53-mediated transcriptional activation. Oncogene. 1995; 10:1275-1282.

13. Haupt Y, Maya R, Kaza A, Oren M. Mdm2 promotes the rapid degradation of p53. Nature. 1997; 387:296-299.

14. Kubbutat MHG, Jones SN, Vousden KH. Regulation of p53 stability by Mdm2. Nature. 1997; 387:299-303.

15. Honda R, Tanaka H, Yasuda Y. Oncoprotein MDM2 is a ubiquitin ligase E3 for tumor suppressor p53. FEBS Lett. 1997; 420:25-27.

16. Montes De Oca Luna R, Wagner DS, Lozano G. Rescue of early embryonic lethality in mdm2-deficient mice by deletion of p53. Nature. 1995; 378:203-206.

17. Jones SN, Roe AE, Donehower LA, Bradley A. Rescue of embryonic lethality in Mdm2-deficient mice by absence of p53. Nature. 1995; 378:206-208.

18. Balint E, Bates S, Vousden KH. Mdm2 binds p73 without targeting degradation. Oncogene. 1999; 18:3923-3929.

19. Zeng $X^{1}$, Chen L, Jost CA, Maya R, Keller D, Wang X, Kaelin WG Jr, Oren M, Chen J, Lu H. MDM2 suppresses p73 function without promoting p73 degradation. Mol Cell Biol. 1999; 19:3257-7366.

20. Leng RP, Lin Y, Ma W, Wu H, Lemmers B, Chung S, Parant JM, Lozano G, Hakem R, Benchimol S. Pirh2, a p53 induced ubiquitin-protein ligase, promotes p53 degradation. Cell. 2003; 112:779-791.

21. Wu H, Pomeroy SL, Ferreira M, Teider N, Mariani J, Nakayama KI, Hatakeyama S, Tron VA, Saltibus LF, Spyracopoulos L, Leng RP. UBE4B promotes Hdm2mediated degradation of the tumor suppressor p53. Nature Medicine. 2011; 17:347-356.

22. Wu H, Zeinab RA, Flores ER, Leng RP. Pirh2, a ubiquitin E3 ligase, inhibits p73 transcriptional activity by promoting its ubiquitination. Mol Cancer Res. 2011; 9:1780-1790.
23. Honda R, Yasuda H. Activity of MDM2, a ubiquitin ligase, toward p53 or itself is dependent on the RING finger domain of the ligase. Oncogene. 2000; 19:1473-1476.

24. Fang S, Jensen JP, Ludwig RL, Vousden KH, Weissman AM. Mdm2 is a RING finger-dependent ubiquitin protein ligase for itself and p53. J Biol Chem. 2000; 275:8945-8951.

25. Li M, Brooks CL, Wu-Baer F, Chen D, Baer R, Gu W. Mono- versus polyubiquitination: differential control of p53 fate by Mdm2. Science. 2003; 302:1972-1975.

26. Zhao M, Zhang NY, Zurawel A, Hansen KC, Liu CW. Degradation of some polyubiquitinated proteins requires an intrinsic proteasomal binding element in the substrates. J Biol Chem. 2010; 285:4771-4780.

27. Kubo N, Okoshi R, Nakashima K, Shimozato O, Nakagawara A, Ozaki T. MDM2 promotes the proteasomal degradation of $\mathrm{p} 73$ through the interaction with Itch in HeLa cells. Biochem Biophys Res Commun. 2010; 403:405-411.

28. Kawai H, Wiederschain D, Yuan ZM. Critical contribution of MDM2 acidic domain to p53 ubiquitination. Mol Cell Biol. 2003; 23:4939-4947.

29. Meulmeester E, Frenk R, Stad R, de Graaf P, Marine JC, Vousden KH. Critical role for a central part of Mdm2 in the ubiquitylation of p53. Mol Cell Biol. 2003; 23:4929-4938.

30. Sharma S, Schwarte-Waldhoff I, Oberhuber H, Schafer R. Functional interaction of wild-type and mutant p53 transfected into human tumor cell lines carrying activated ras genes. Cell Growth Differ. 1993; 4:861-869.

31. Fang L, Lee SW, Aaronson SA. Comparative analysis of p73 and p53 regulation and effector functions. J Cell Biol. 1999; 147:823-830.

32. Lau LM, Nugent JK, Zhao X, Irwin MS. HDM2 antagonist Nutlin-3 disrupts p73-HDM2 binding and enhances p73 function. Oncogene. 2008; 27:997-1003.

33. Busuttil V, Droin N, McCormick L, Bernassola F, Candi E, Melino G, Green DR. NF-kappaB inhibits T-cell activationinduced, p73-dependent cell death by induction of MDM2. Proc Natl Acad Sci USA. 2010; 107:18061-18066.

34. Hershko A, Ciechanover A. The ubiquitin system for protein degradation. Annu Rev Biochem. 1992; 61:761-807.

35. Pickart CM. Ubiquitin in chains. TIBS. 2000; 25:544-548.

36. Hicke L. Protein regulation by monoubiquitin. Nat Rev Mol Cell Biol. 2001; 2:195-201.

37. Finley D, Sadis S, Monia BP, Boucher P, Ecker DJ, Crooke ST, Chau V. Inhibition of proteolysis and cell cycle progression in a multiubiquitination-deficient yeast mutant. Mol Cell Biol. 1994; 14:5501-5509.

38. Sigismund S, Polo S, Di Fiore PP. Signaling through monoubiquitination. Curr Top Microbiol Immunol. 2004; 286:149-185. 